\title{
Innovative Solution for Harvesting Energy in Marine Vessels
}

\author{
George Nerubenko ${ }^{1}$, Dmytriy Gurevych ${ }^{2}$ \\ ${ }^{1}$ NER*MAR Limited \\ 270 Palmdale Drive, Toronto, ON, M1T 3N8 Canada \\ optimalproject@hotmail.com \\ ${ }^{2}$ Mega Automation inc \\ 273 Bowes road, Concord, ON, L4K 1H8, Canada \\ dgurevych@mega-automation.ca
}

\begin{abstract}
The marine vessels are subjected to various aggressive dynamic loads. Mainly they cause the roll, pitch, heave and yaw of marine vessels.One of the recently invented effective tools for suppressing the negative impact of these loads is the energy harvesting devices. The proposed energy harvester consists of two main components: tuned mass damper and electricity generator. The innovative part of proposed energy harvester is the introduction of control system into structure of tuned mass damper providing the automatic adjustment of springstiffness coefficient in accordance to the current frequency of external force. Such energy harvesting devices are targeting two issues: reduction of impacts of dynamic loads acting on vessel and obtaining additional portion of electrical energy for free. The light boat is selected as an example for demonstration of all steps of energy harvesting technology implementation into marine practice.
\end{abstract}

Keywords: Vibration energy harvesting damper, Electricity generator, Pitching boat.

\section{Introduction}

The marine vessels are experiencing different types of dynamic loads during operation [1,2]. One of the results of dynamic loads is the various kinds of vessel's oscillations. The authors selected one type of oscillations known as "pitching" for study in that paper. In pitching the marine vessel is lifted at the bow and lowered at the stern and vice versa. It is obvious that such oscillations have the negative impact on all components of ship and crew operations. The practice demonstrates [3] that the frequencies of above mentioned disturbances are sitting mainly in range $0.0 \mathrm{~Hz}-3.0 \mathrm{~Hz}$. One of the most effective instruments for suppressing such oscillations is Tuned Mass Damper (TMD) - the device consisting of mass reacting on oscillating object movements through specifically designed springing element [4]. The idea of combining the damping properties of TMD with energy harvesting was developed recently in [5,6] and that device was called the Vibration Energy Harvesting Damper (VEHD). The energy harvesting is the technology allowing capture, convert and transmit the certain amount of wasted power [7,8]. The proposed VEHD could be manufactured in two main versions: for rectilinear vibration applications and for angular rotational applications. The results of VEHD development for different industries were discussed in $[9,10]$. The implementation of VEHD in marine vessels is dictated by two interconnected reasons: necessity of a reduction of intensive vessel oscillations and proper usage of damped vibration energy. The current study is the attempt to introduce the VEHD technology into marine vessels. The light boat is selected as an object for study.

\section{The model of pitching boat equipped byVEHD}

The light boat scheme is presented in Figure 1.The boat is in calm deep water. Assume that boat's body is a solid object moving with stationary speed, and no waves induced. Unmovable system of coordinate is X0Z, and X0Z plane is coincided to Figure 1 plane. Centre of vessel mass $\mathbf{M}$ is in 0. Mass moment of vessel's inertia relatively to axis $0 \mathrm{Y}$ (perpendicular to Figure 1 plane) is equal to $\mathbf{J}$. Considered small oscillations $\boldsymbol{\theta}$ are in $\mathrm{XOZ}$ plane around 0 transverse axes. The restoring moment of forces $\mathbf{c} \boldsymbol{\theta}$ and moment of resistance forces $\boldsymbol{\beta} \boldsymbol{\theta}$ are applied during pitching. The $\mathbf{c}$ and $\boldsymbol{\beta}$ are the coefficients of restoring moment and viscous friction.

The presence of wave loads would be modelled by member Mo $\sin \left(2 \pi t / T_{0}\right)$, where Mo is the external moment of forces and To $=2 \pi / \omega$ is wave period and $\omega$ is the frequency. Suppose that parameters of a boat are $\mathbf{J}=2.49 \times 10^{3} \mathrm{kgm}^{2}, \mathbf{c}=1.15 \mathrm{x}$ 
$10^{5} \mathrm{kgm}^{2} / \mathrm{s}^{2}, \boldsymbol{\beta}=2300 \mathrm{kgm}^{2} / \mathrm{s}^{2}$, and the mass of the boat is $\mathbf{M}=24.62$ ton. Thinking about the dynamics of boat pitch it is reasonable to propose the symmetric mounting of two rectilinear VEHD (one at the nose area and other at the stern). The core graphical model of rectilinear operating VEHDis shown in Figure 2.

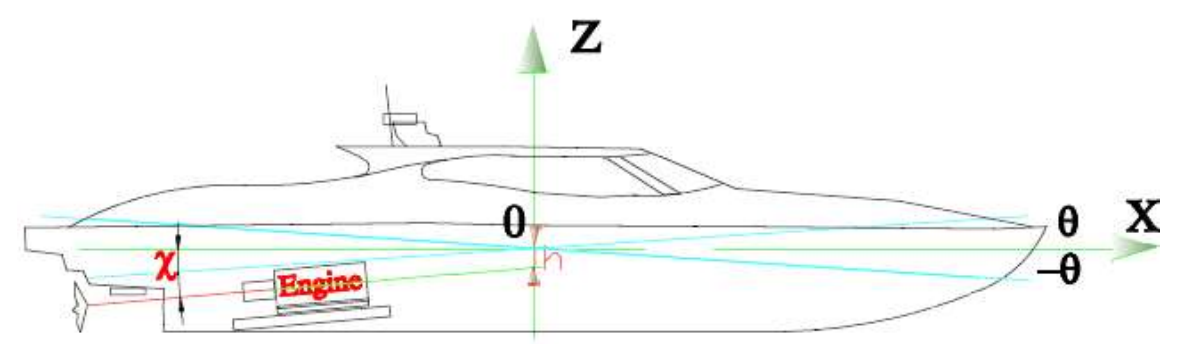

Fig. 1: Light boat general scheme.

First of all it is necessary to underline that local coordinate system in Figure 2 is used for point on a boat where VEHD is attached.

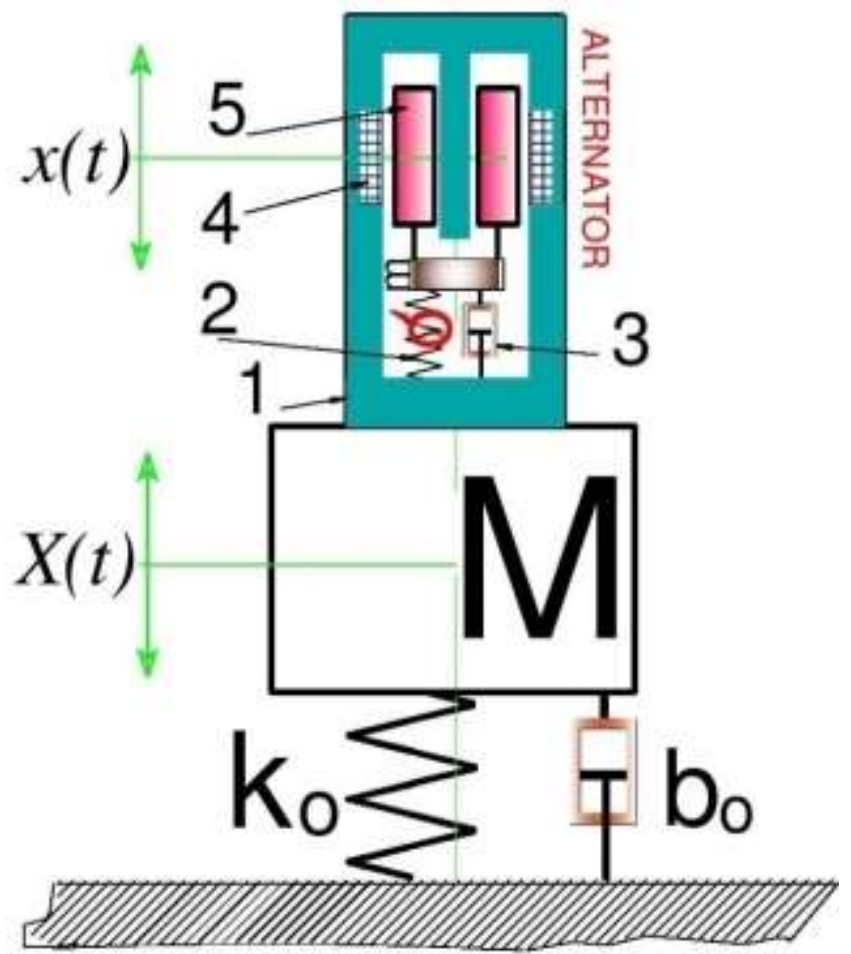

Fig. 2: VEHD arrangement for one-dimension application.

Let's assume that an object (for instance stern) is oscillating approximately along coordinate axis $\mathbf{X}$ as shown in Figure 2. The body 1 of VEHD is attached firmly to object. VEHD consists of two major parts: tuned mass damper and alternator. TMD having mass $\mathbf{m}$ is attached to VEHD body by means of spring 2 and damping element 3 . Alternator part consists of pick-up coil 4 and magnet 5 (for instance, configured as a ring). The coil 4 is embedded or otherwise secured within the tuned mass $\mathbf{m}$ in a position surrounding the magnet 5 and terminates in a pair of electrical contacts (not shown in Figure 2), which may be connected to the power system or other electrical load. Magnet 5 is attached firmly to the mass $\mathbf{m}$ of tuned mass damper. Tuned mass damper has to have own resonant frequency equal to a boat pitch frequency. The amplitude of mass $\mathbf{m}$ vibrations would be maximal in this case, and the magnitude of produced electricity in alternator would be the maximal as well. It is assumed (for simplicity of a model), that the mono harmonic low frequency (up to $3 \mathrm{~Hz}$ ) pitch is applied to the boat body. Let's suppose further that the object' properties are modeling by spring having stiffness $\mathbf{k o}=\mathbf{c}$ and viscosity factor $\mathbf{b o}=\boldsymbol{\beta}$. Let's consider that it would be reciprocal movements in one 
direction only. Its movement coordinate is $\mathbf{X}(\mathbf{t})$, where $\mathbf{t}$ is time. The outer casing of VEHD is firmly attached to the object, and the mass of casing is included in $\mathbf{M}$. Tuned mass damper $\mathbf{m}$ is connected to casing by means of springs having the stiffness $\mathbf{k 1}$ and viscosity factor $\mathbf{b 1}$. Mass $\mathbf{m}$ is vibrating in the same direction as mass $\mathbf{M}$. The coordinate of vibrations of $\mathbf{m}$ is $\mathbf{x}(\mathbf{t})$. It is shown in [9], [10] that the highest effectiveness of VEHD could be reached when $\mathrm{k} 1 / \mathrm{m}=\omega_{\mathrm{o}}^{2}$ is adjusted to variable external force frequency $\omega$, or $\mathrm{k} 1 / \mathrm{m}=\omega^{2}$, however it must be used the special control device in this case providing metering of the changing values $\omega$, and adjusting stiffness coefficient of spring $\mathrm{k} 1$. In that case the magnitudes of k1 would be variable. The electromagnetic subsystem is inducing current $\mathbf{i}$, and $\mathbf{L}$ is inductance, $\mathbf{R}$ is electrical resistance, where $\mathbf{R c}$ is coil resistance and $\mathbf{R d}$ is load resistance, and $\mathbf{R}=\mathbf{R} \mathbf{c}+\mathbf{R d}$. The VEHD dynamics is described by system of ordinary differential equation (see [7-11], [14,15])

$$
\begin{aligned}
& \mathbf{J} \theta^{\prime \prime}+\beta \theta^{\prime}+\mathbf{c} \theta+\mathbf{m} \omega^{2}(\theta-x / r)+b_{1}\left(\theta^{\prime}-x^{\prime} / r\right)=\operatorname{Mosin}(\omega t) \\
& \mathbf{m x}{ }^{\prime \prime}-\mathbf{m} \omega^{2}(\theta-\mathrm{x} / \mathrm{r})-\mathbf{b}_{1}\left(\boldsymbol{\theta}^{\prime}-\mathrm{x}^{`} / \mathrm{r}\right)+\Phi \mathbf{m i}=0 \\
& \mathbf{L i}+\mathbf{R i}-\boldsymbol{\Phi e}\left(\mathrm{x}^{`}-\mathrm{r} \boldsymbol{\theta}^{\prime}\right)=0
\end{aligned}
$$

where ${ }^{\prime}=\mathrm{d} / \mathrm{dt}, \boldsymbol{\Phi m}$ is the linkage factor between mechanical and electromagnetic subsystems in mechanical terms, $\Phi \mathbf{e}$ is the linkage factor between electromagnetic and mechanical subsystems in electromagnetic terms, and $\mathbf{r}$ is the distance from a centre of vessel mass to VEHD location.

The system of equations (1) - (3) includes the specific features like automatic frequency tuning $\omega_{\mathrm{o}}=\omega_{\text {, and }}$ the link between vibrations of Tuned Mass Damping subsystem and dynamic behaviour of current generating subsystem (equation (3)). It gives the possibility pay attention on the dynamic properties of TMD coupled to an alternator.

\section{The dynamical and energy features of VEHD installed on pitching boat}

Selecting for considered example the boat parameters: $M=24620 \mathrm{~kg}, \mathrm{bo}=2300 \mathrm{kgm}^{2} / \mathrm{s}, \mathrm{ko}=1.1 .5^{*} 10^{5} \mathrm{kgm}^{2} / \mathrm{s}^{2}, \mathrm{~J}=2490$ $\mathrm{kgm}^{2}$, external load $-\mathrm{Mo}=2100 \mathrm{kgm}^{2} / \mathrm{s}^{2}$ (heavy wave load), and using the equation (1) it is possible to build the amplitude frequency characteristic (green solid curve) for boat as shown in Figure 3. The abscissa horizontal axis is for excitation frequency in $\mathrm{Hz}$ in these Figures (further similar on following Figures) and ordinate vertical axis is for amplitude (m) in Figure 3.

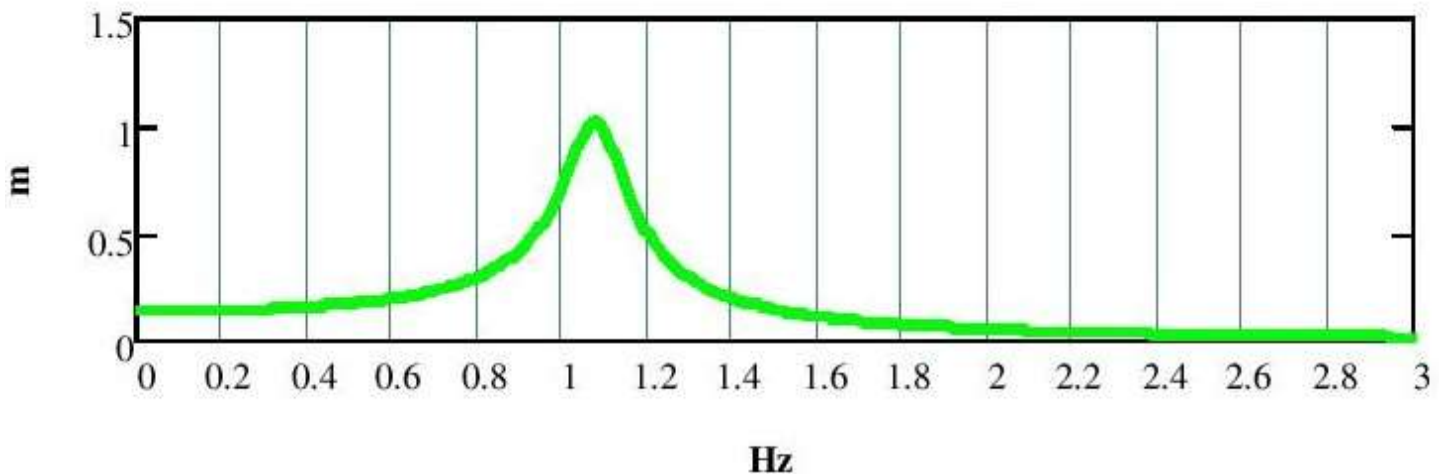

Fig. 3: Dynamical characteristics for examined boat.

Analyzing the amplitude - frequency characteristic in Figure 3, one can see that peak of the amplitude of $1.023 \mathrm{~m}$ is registered at frequency $1.08 \mathrm{~Hz}$; it means that the pitch resonance could occur at frequency $1.08 \mathrm{~Hz}$ for considered boat. Hence the boat will be experiencing the significant pitching at $1.08 \mathrm{~Hz}$ if there are no additional devices installed on that boatfor suppressing the negative impact of wave load.

Prior to examination of installation of VEHD into boat structure let's formulate the purpose of this installation. The main target of design of proposed VEHD is to satisfy two conditions: a. generation of an additional electrical energy which could be used, b. a reduction of the pitch levels.

There is no information about VEHD parameters at this point, so suppose that the total mass of TMD portion is selected equal to $0.2 \%$ of boat's mass following the minimal additional weight requirements. The installed system plans to be presented by twin VEHD, first VEHD is located on bow and second VEHD is on stern. The distance r=7.6 m, the weight of 
TMD portion in each VEHD is $24.62 \mathrm{kG}$. Keep in mind that $\mathrm{k} 1 / \mathrm{m}=\omega^{2}$. Let's assume for simplicity that the initial parameters of an alternator are selected following [9,10] recommendations. Using the system of differential equations (1) - (3) the amplitude - frequency characteristics of considered boat equipped by VEHD could be built (see Figure 4A). A green curve reflects the vibration behaviour of pitching boat without installed VEHD, and this curve coincides to green curve in Figure 3. The blue dotted curve in Figure 4A stands for damped vibrations of pitching boat when VEHD is installed. The maximum damped amplitude (according to blue dotted line) is $0.476 \mathrm{~m}$, so the reduction of resonance amplitude is in 2.15 times, which is sufficient for examined situation.

VEHD peak of useful power could be plotted by solid magenta curve (see Figure 4B). VEHD useful power is calculating by formula $\mathbf{P}(\omega)=0.5 \mathbf{R d} \mathbf{i}^{2}$, where $\mathbf{R d}=0.5 \mathbf{R}$. The peak of useful power is $1.1 \mathrm{~kW}$.
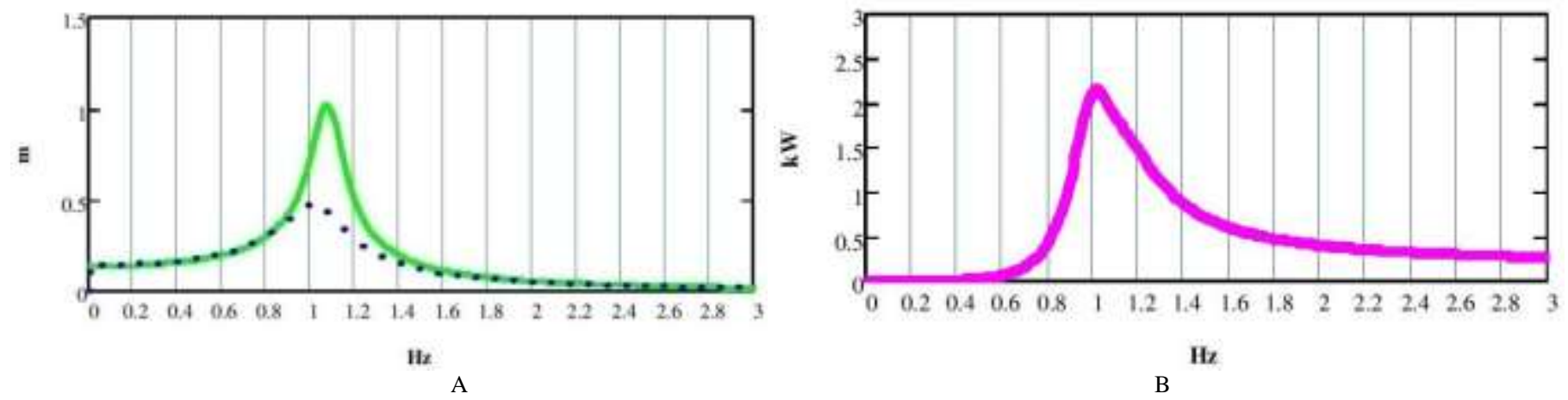

Fig. 4: Dynamical and power characteristics for boat when VEHD having $0.2 \%$ of boat's mass installed.

Let's analyze the case when the lighter VEHD is used. Assume that the total mass of TMD portion is selected equal to $0.1 \%$ of boat mass. There are the results of model simulation for such case in Figure 5. The amplitude - frequency characteristics of boat equipped by lighter VEHD is shown in Figure 5A by blue dotted curve.
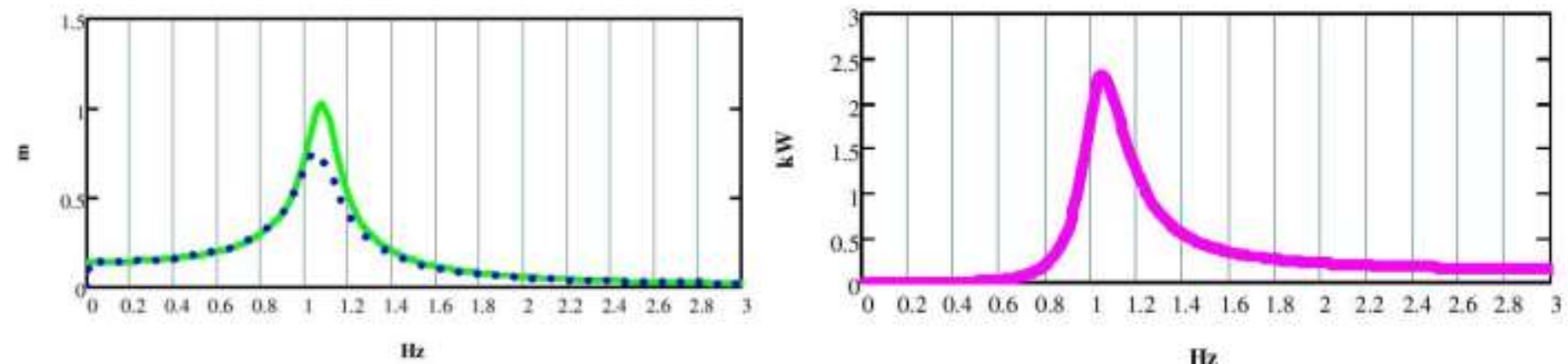

Fig. 5: Dynamical and power characteristics for boat when VEHD having $0.1 \%$ of boat's mass installed.

The maximum damped amplitude (according to blue dotted line) is $0.751 \mathrm{~m}$, so the reduction of resonance amplitude is in 1.36 times, which is less than for previous case when mass of VEHD is $0.2 \%$. Actually that result is obvious - the reduction of TMD weight leads to reduction of suppression efficiency. The maximal value of a peak of useful power is $2.3 \mathrm{~kW}$. The comparison to previous case illustrates the significant increase of generated power (in 2.09 times). It is necessary to note that reduction of TMD weight is restricted by geometrical conditions; hence it is practically impossible to use the VEHD with $0.08 \%$ of boat mass.

This cycle of study indicates that the engineer has some options in a process of VEHD parameter's selection. It is possible install heavier VEHD for obtaining the better results in damping of the boat pitch, and in this case the effectiveness of usage of wasted energy (converting pitch energy to electricity) would be reduced. From the other hand, it is possible install lighter VEHD for obtaining the better results in converting pitch energy to electricity, however the effectiveness in damping of the boat pitch would be reduced. 


\section{VEHD tests}

The special swinging boat model was designed for modelling tests in Ukrainian ship model basin. The design of the model was similar to ideas discussed in [16]. That boat model has a mass equals to 1:100 of real boat mass $246 \mathrm{~kg}$. When the right end of model is up - the left end of model is down. The two of water-proof prototypes of VEHD were designed (based on above presented analysis) and fabricated. The movable mass of VEHD twin prototype is $0.246 \mathrm{~kg}$. It was designed and manufactured the control system providing automatic tuning and synchronizing of VEHD operation and extracted energy storage.

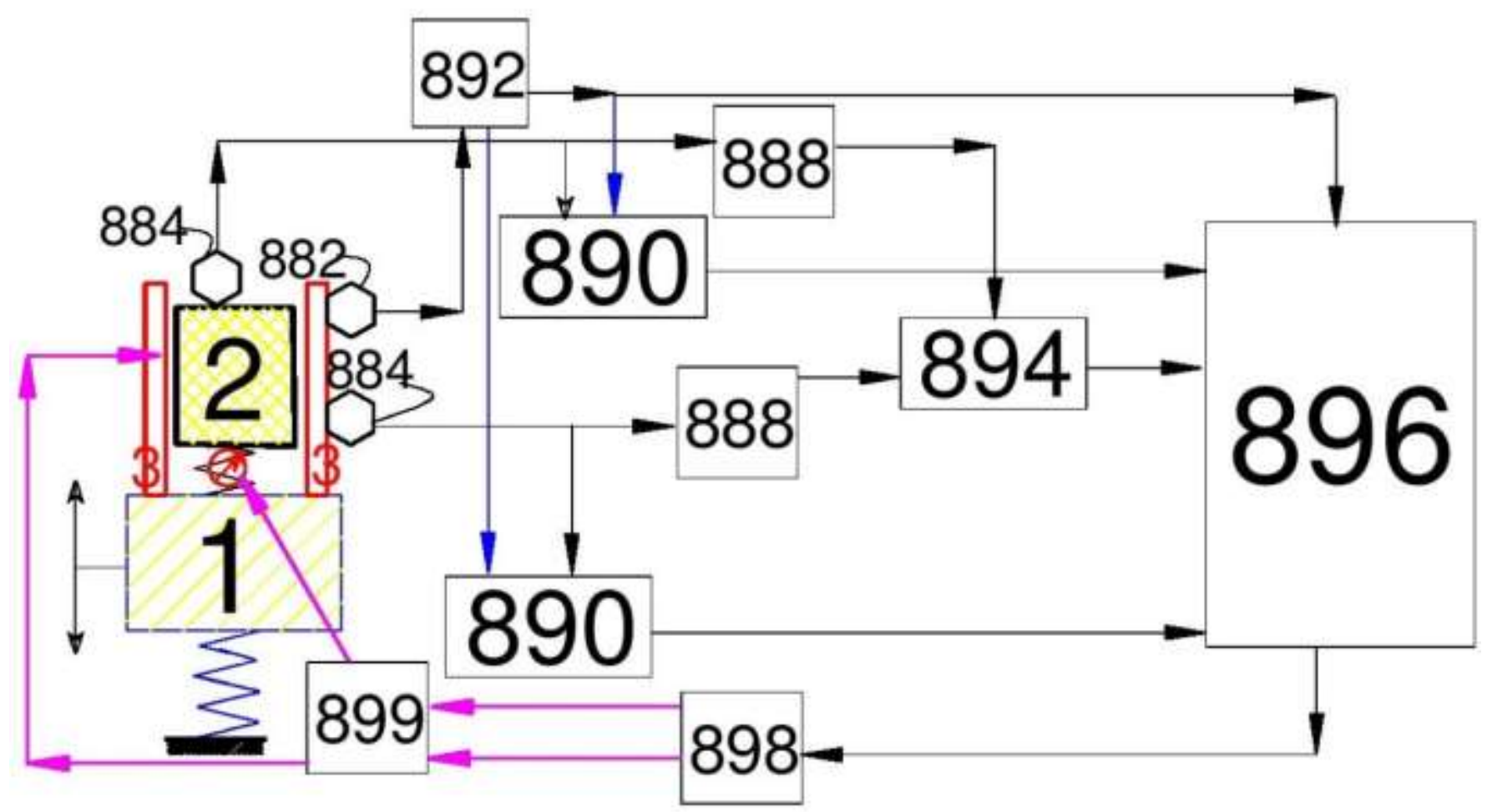

Fig. 6: Schematic diagram of VEHD spring stiffness control.

Figure 6 shows the proposed versions of the schematic diagram of VEHD spring stiffness control by electromagnetic apparatus 800. Accelerometers $\mathbf{8 8 4}$ are connected a. to 2 - TMD and b. to the 3 - electromagnetic solenoid(shown in cross section). The accelerometers $\mathbf{8 8 4}$ are each connected to integrators $\mathbf{8 9 0}$. Both outputs of integrator $\mathbf{8 9 0}$ are connected to computing system 896. The accelerometers $\mathbf{8 8 4}$ are also connected to according phase detectors $\mathbf{8 8 8}$ that are, in turn, connected to one phase discriminator 894. This is, in turn, connected to the computing system 896. Also connected to the solenoid is a frequency detector $\mathbf{8 8 2}$ that is connected, via filter 892, to the integrators 890 and the computing system $\mathbf{8 9 6}$. The filters and amplifiers may be used, where appropriate to ensure proper signal transmission. The computing system is connected to a signal generator $\mathbf{8 9 8}$, which is connected to the actuator of springing element (located between 1 and 2) and to solenoid 3. The computing system $\mathbf{8 9 6}$ has the capacity to (1) input frequency, amplitude and phase difference data, (2) perform algorithms on this data, and (3) generate command signals to govern VEHD. The computing system 896 accommodates the designed algorithm, which provides self-tuning. Solenoid $\mathbf{3}$ is connected (not shown) to the on-board storage.

The photo of the test rig system control box is shown in Figure 7. 


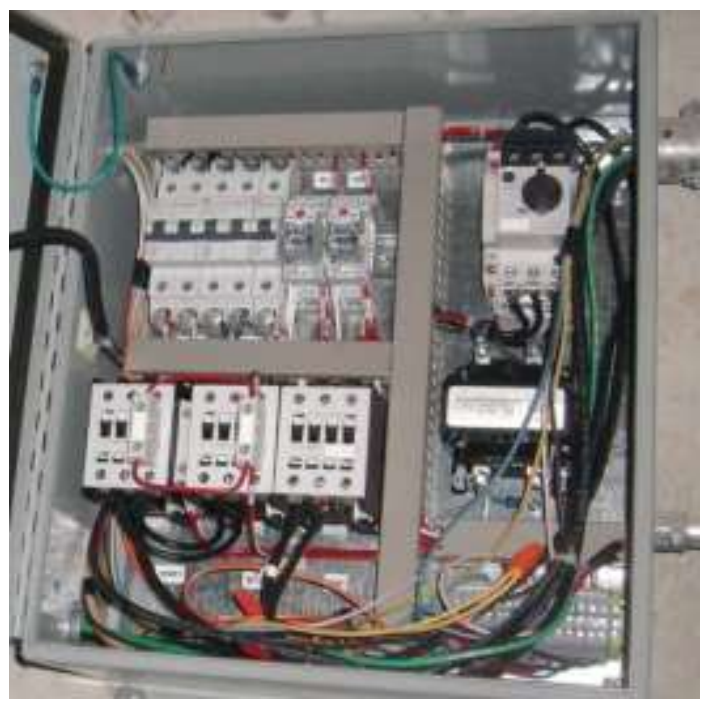

Fig. 7: Test rig system control box.

VEHD were vertically installed. There was a specifically designed shaker used for swinging experiments modeling the pitching boat behaviour.The shaker has the driving device in form of DC Motor, electrically connected to speed controller for controlling DC RPM. The DC Motor is connected to system of gears for getting low frequency model excitation (in range $0-5 \mathrm{~Hz})$.
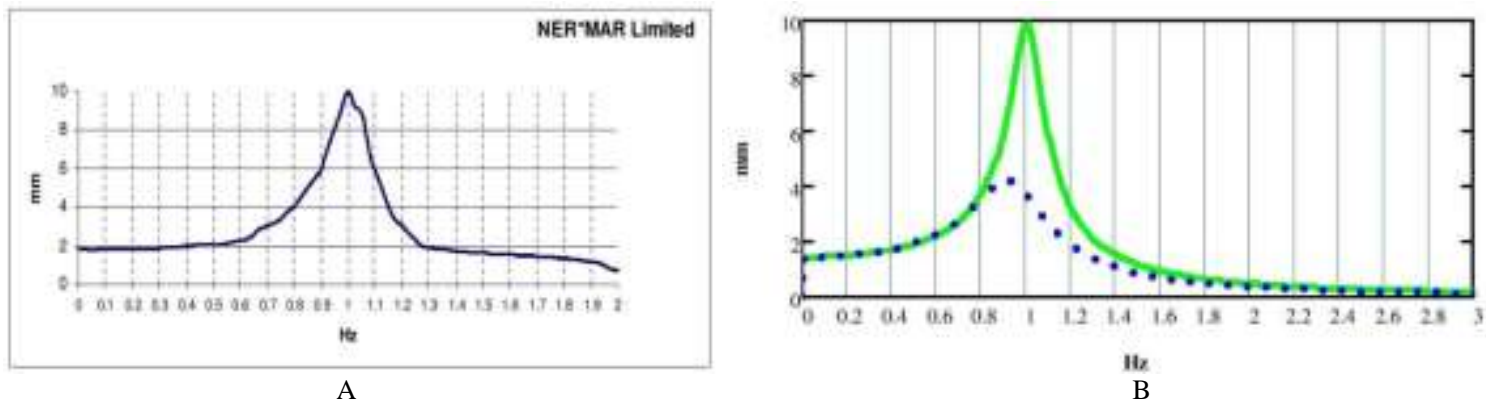

Fig. 8: Real (A) and modeled (B) dynamical behavior of basin boat model.

First of all the amplitudes of swinging boat model were recorded at location of projected VEHD installation. The range of excitation frequencies was $0-2 \mathrm{~Hz}$. The results of measurements are presented in Figure $8 \mathrm{~A}$ where the horizontal axis stands for frequencies in $\mathrm{Hz}$, and vertical axis stands for amplitudes in $\mathrm{mm}$. The recordings show that there is one resonance peak at $1.0 \mathrm{~Hz}$, and maximum amplitude value is $9.98 \mathrm{~mm}$. Remembering the accepted scale 1:100 one can state the satisfactory correlation between boat model data and real boat (object) data.

The next step was to adjust and tune the VEHD parameters to accepted model. It was built the mathematical model based on system (1) - (3) and the procedure of adjustment was used to be sure that mathematical model coincided and reflected the behaviour of basin boat model. The result of that procedure is shown graphically in Figure $8 \mathrm{~B}$. The data names presented in Figure 8B are the same as in Figures 4A and 5A. The green solid curve reflects the dynamical behaviour of basin boat model without VEHD. The resonance peak is $9.9 \mathrm{~mm}$ at $1.0 \mathrm{~Hz}$. The shape of given amplitude - frequency characteristic coincides practically to experimentally obtained one in Figure 8A. These results allow to tune and properly adjust the VEHD. The expected amplitude - frequency characteristic of a basin boat model with VEHD installed is shown by blue-dotted curve in Figure 8B. The expected maximum amplitude of platform would be $4.2 \mathrm{~mm}$.

The planned VEHD peak of useful power is plotted by solid magenta curve in Figure 9. It is needed to highlight that the curve has two peaks, and one minimum between these two peaks. The above mentioned minimum is located at $1 \mathrm{~Hz}$; this is typical dynamical picture when TMD is installed. The theoretical maximum value of useful power is equal 
to $0.21 \mathrm{~kW}$; however it must be stated that additional free generating power could be obtained in broad interval of frequencies.

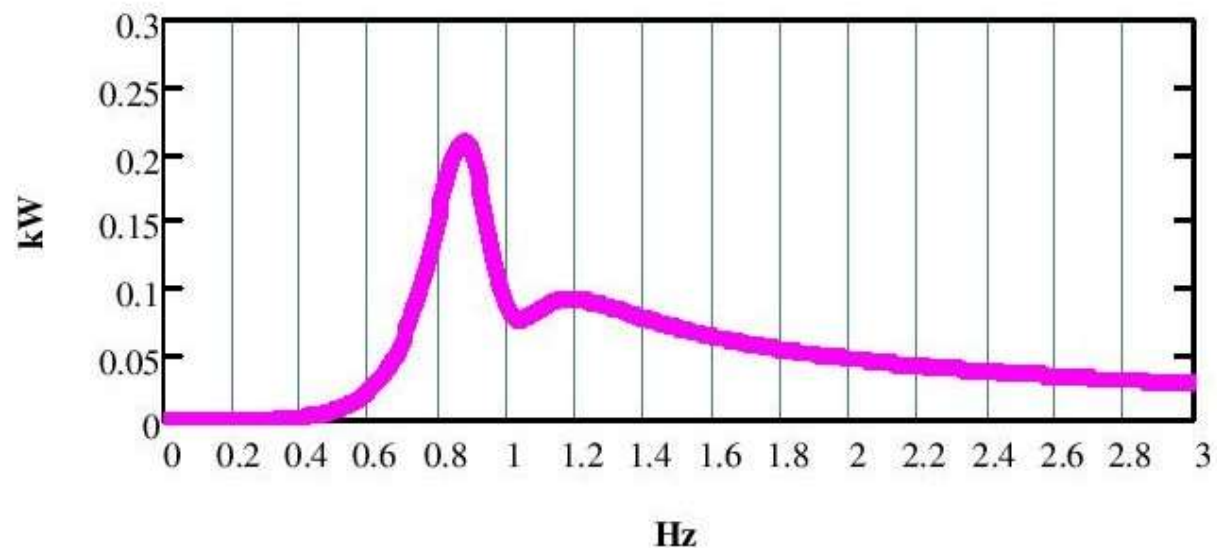

Fig. 9: VEHD peak of useful power (theory).

The real dynamical behaviour of tested basin boat model equipped by VEHD was recorded (see Figure 10). There is one peak only of $4.8 \mathrm{~mm}$ at $1.0 \mathrm{~Hz}$. The measured maximum value of useful power is equal to $0.20 \mathrm{~kW}$.

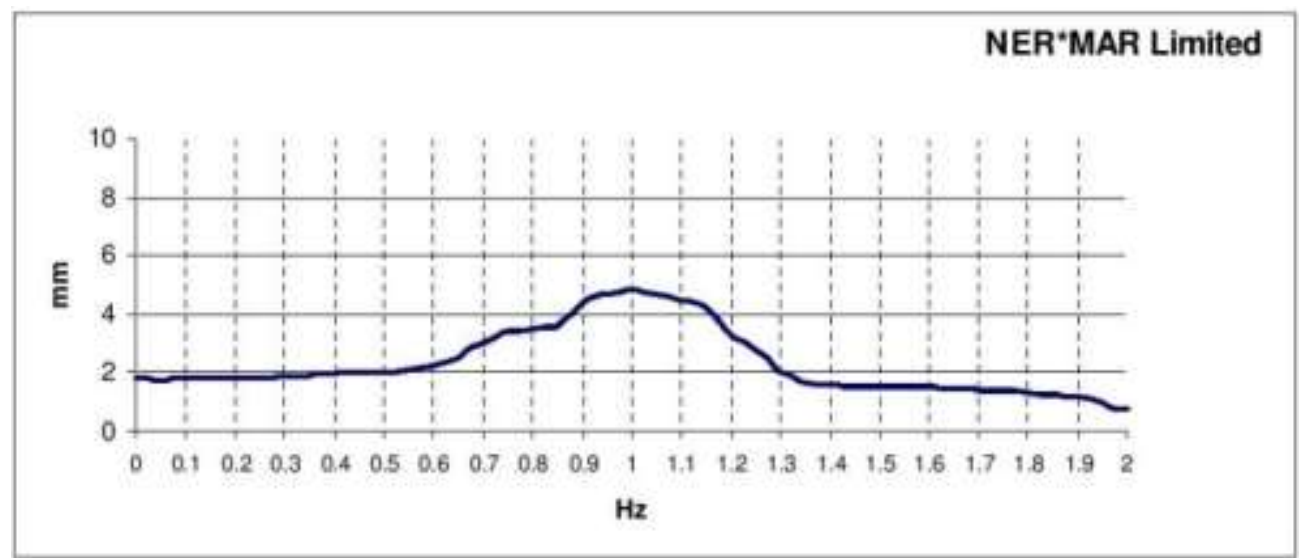

Fig. 10: The recorded dynamical behaviour of basin boat model when VEHD installed.

The comparison of values metered during tests to data received theoretically shows that the deviation of obtained data from theoretically predicted is small; and it could be concluded that these results confirm a satisfactory compliance of dynamic and energy properties of real fabricated VEHD to theoretically predicted model.

\section{Conclusion}

The harsh dynamical problems of marine vessel operation can be successfully solved using invented schemes of energy harvesting emissions-free devices. The main purpose of design and implementation of proposed energy harvesting tools is to serve dual goals:

- reduction of the all kinds of dynamic loads (including pitch, roll and yaw) of a vessel by controlled tuned mass dampers included in proposed energy harvesting devices,

- generating an additional portion of power which could be directed to on-board energy storage system or for other applications.

There are a lot of positive side effects of introducing invented energy harvesting devices such as reduction of fatigue in vessel structures, comfort level improvements, feeding wireless autonomous electronic units and so on.

The presented study confirms the effectiveness of invented vibration energy harvesting damper for suppressing the marine vessels pitch. Based on a provided dynamical analysis it could be recommended to use the system of vibration energy 
harvesting dampers having total movable mass of tuned mass damper's portion equal to $0.2 \%$ of vessel's mass. It gives the opportunity to obtain the pitch amplitude lowering in more than 2 times accompanied by free generation of electrical power. The reduction of the movable mass of tuned mass damper's portion to values less than $0.2 \%$ of vessel's mass leads to worst results in the pitch amplitude lowering and cannot be recommended.

The developed energy harvesters could be installed and used successfully in various types of marine vessels allowing generates for free the significant amounts of energy.

\section{Acknowledgements}

A special thanks to Vice President of National University of Shipbuilding (Nikolaev, Ukraine) Edward Khachaturov Professor, Doctor of Sciences, PH. D. for the valuable help during experiments.

\section{References}

[1] EdwinKreuzer, Marc-Andre Pick, "Dynamics of Ship-Motion," PAMM • Proc. App. Math. Mech., vol. 3, pp. 84-87, 2003.

[2] J. Matusiak, "Dynamics of a Rigid Ship," Aalto University Publication Series Science + Technology, p. 156, 2013.

[3] L. H. J. Holthuijsen, Waves in Oceanic and Costal Waters. Cambridge: Cambridge University Press, 2007.

[4] Sergey Eliseev, George Nerubenko, "Tuned Mass Dampers," NAUKA, Novosibirsk, 1982.

[5] George Nerubenko, "Torsional Vibration Damper of a Rotating Shaft," US Patent 7464 800, December 16, 2008.

[6] George Nerubenko, et al., "Vibration Energy Harvesting Damper," U.S. Patent Application No. 16119 346, August 31, 2018.

[7] S. Priya, and D. L. Inman, Energy Harvesting Technologies. Sprinder, Verlag, 2009.

[8] Francesco Cottone, "Energy Harvesting: Introduction," NiPS Summer School 2015 - July 7-12 th Fiuggi, Italy, p. 50.

[9] Alexander Bograd, George Nerubenko, Cyril Nerubenko, "Energy Harvesting in Vehicle's Drive," Proceedings of the 2nd International Conference of Energy Harvesting, Storage, and Transfer (EHST'18), Niagara Falls, Canada, June 7 9, 2018, no. 112.

[10] George Nerubenko, et al, "Tuned mass dampers as energy harvesters for railways," Proceedings of ISMA2018 and USD2018, Leuven, Belgium, pp. 1689-1702.

[11] Vladimir Babitsky, George Nerubenko, "About One Case of Excitation in System of Vessel and Propulsive Complex," Machine Science, no. 6, pp. 3-6, 1980.

[12] Francesco Cottone, Suresh Goyal, Jeff Punch, "Energy harvester apparatus having improved efficiency," US Patent 8350 394, January 8, 2013.

[13] Roy Freeland, Stephen Roberts, "Electromechanical generator for converting mechanical vibrational energy into electrical energy, US Patent 7999 402, August 16, 2011.

[14] Hongxin Sun, Yifan Luo, Xiuyong Wang, Lei Zuo, "Seismic control of a SDOF structure through electromagnetic resonant shunt tuned mass-damper-inerter and the exact H2 optimal solutions," Journal of Vibroengineering, vol. 19, no. 3, pp. pp.2063 - 2079, 2017.

[15] Daqaq S. V. M. F. et al., "Investigation of Power Harvesting via Parametric Excitations," Journal of Intelligent Material Systems and Structures, vol. 20, no. 5, pp. 545-557, 2009.

[16] Richard A. Royce, "Ship model testing.” Final report. N00014-10-1-0773 Web Institute, p. 15, 2016. 